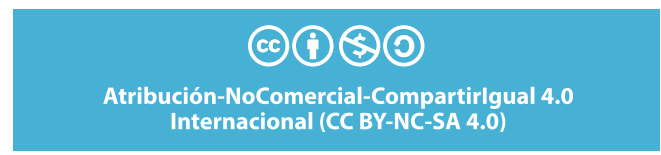

DOI: http://dx.doi.org/10.20983/reij.2022.1.4
Débora Mariel Etchegaray ${ }^{1}$

Clara María Minaverry ${ }^{2}$

FECHA DE RECEPCIÓN: 14 DE JULIO 2021

FECHA DE ACEPTACIÓN 08 DE SEPTIEMBRE 2021

\title{
ANÁLISIS COMPARATIVO DEL MARCO JURÍDICO FORESTAL Y DE LOS SERVICIOS ECOSISTÉMICOS EN ARGENTINA, COLOMBIA Y PERÚ
}

\author{
Comparative analysis of forestry legal framework and \\ ecosystems services in Argentina, Colombia and Peru
}

\section{Resumen}

El presente trabajo tiene como objetivo realizar un análisis del marco jurídico forestal y de los servicios ecosistémicos en Argentina (a nivel nacional), y efectuar un análisis comparativo con la situación jurídica actual en Colombia y Perú. Se llevó a cabo una estrategia de investigación multimétodo, a través de la observación documental, método de estudio de casos, método de análisis comparativo-descriptivo y modelo de guía de análisis. En cuanto a los resultados obtenidos, a partir del análisis comparativo se obtuvieron fortalezas y debilidades. Por un lado, los tres casos buscan el uso sostenible y conservación de los bosques; no obstante, se observó una profusa normativa fragmentada que regula solo aspectos parciales de los bosques. Finalmente, a modo de conclusión, se destaca que los bosques nativos poseen un gran valor para la biodiversidad y la vida humana, gracias a todos los servicios/beneficios que estos brindan. Sin embargo, se debe fortalecer la normativa en materia de bosques y de sus servicios ecosistémicos, puesto que en ninguno de los casos se logra una protección jurídica total de estos ecosistemas.

\section{Palabras clave: bosques nativos; servicios ecosistémicos; normativa.}

\section{Abstract}

The present work aims to carry out an analysis of the forest legal framework and ecosystem services in Argentina (at the national level), and to carry out a comparative analysis with the current legal situation in Colombia and Peru. A multi-method research strategy was carried out through documentary observation, case study method, comparative-descriptive analysis method, and analysis guide model. Regarding the results obtained, strengths and weaknesses were obtained from the comparative analysis. On one side, the three cases seek the sustainable use and conservation

1 Licenciada en Información Ambiental; Universidad Nacional de Luján, Argentina. Correo electrónico: dmarieletchegaray@gmail.com. orcid: 0000-0001-8648-6133.

2 Doctora en Derecho; Universidad de Buenos Aires, Argentina. Correo electrónico: clara.minaverry@gmail.com. orcid: https://orcid.org/0000-0003-0153-7224. 
of the forests; however, a profuse fragmented regulation was observed which regulates only partial aspects of the forests. Finally, by way of conclusion, it is highlighted that native forests have great value for biodiversity and human life, thanks to all the services/benefits they provide. However, regulations regarding forests and their ecosystem services must be strengthened since none of the cases have full legal protection of these ecosystems.

Keywords: native forests; ecosystem services; normative.

\section{Introducción}

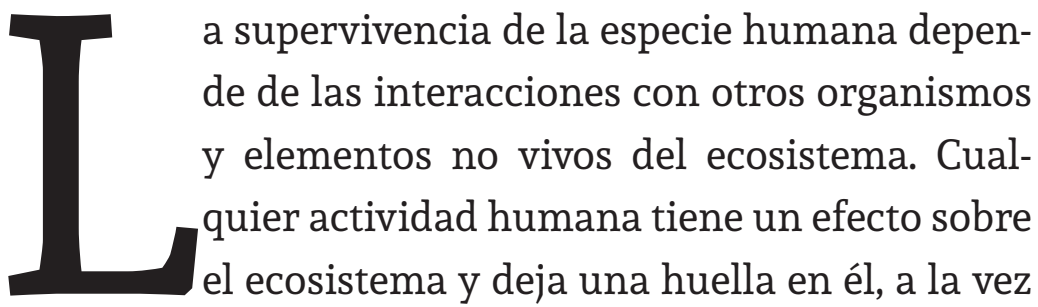
que ejerce una presión sobre el ambiente. Los ecosistemas son vistos como un conjunto de elementos bióticos y abióticos, que interaccionan en un espacio-tiempo y transforman la materia y la energía disponibles en el ambiente mediante procesos funcionales. De estos, se obtienen diferentes beneficios o servicios para el desarrollo de la vida humana, los cuales se conocen como servicios ambientales o servicios ecosistémicos. Según la Evaluación de los Ecosistemas del Milenio, estos beneficios pueden ser económicos, de provisión, de regulación o culturales (Minaverry, 2017).

Los términos "servicios ecosistémicos" y "servicios ambientales" pueden ser utilizados indistintamente, aunque difieren en su contexto. Cuando utilizamos el primero queremos enfatizar el hecho de que el ecosistema es el que permite que los seres humanos se vean beneficiados. En cambio, el término "servicios ambientales" otorga más peso al concepto de "ambiente" o "medioambiente", 
en el cual no se explicitan las interacciones necesarias para proveer dichos servicios (Balvanera, \& Cotler, 2007). No obstante, dentro de este trabajo nos enfocaremos en aquellos servicios ecosistémicos brindados únicamente por los bosques, que resultan ser los ecosistemas terrestres más extensos y que poseen un enorme valor en términos de biodiversidad.

El objetivo de este trabajo será realizar un análisis del marco jurídico forestal y de los servicios ecosistémicos en la República Argentina, a nivel nacional, y efectuar un análisis comparativo con la situación jurídica actual en Colombia y Perú. Y, de esta manera, lograr identificar las fortalezas y debilidades de cada país en torno al marco jurídico que poseen sobre dicha temática.

\section{Metodología}

En el presente proyecto, se implementó la estrategia multimétodo:

a) Observación documental: se obtuvo información mediante la percepción selectiva, ilustrada e interpretativa de un fenómeno determinado. En este caso en particular, se aplicó para el análisis de normas jurídicas, instrumentos internacionales y de políticas públicas vinculada con los bosques nativos y los servicios ecosistémicos.

b) Método de estudio de casos: se implementó con la finalidad de obtener múltiples fuentes de datos e información, como es el caso de las normas jurídicas y de los instrumentos internacionales (al analizarse una selección de países de América del Sur).

c) Método de análisis comparativo-descriptivo: se analizaron normas jurídicas e instrumentos internacionales que difieren entre sí en algunos aspectos (ubicación territorial; contenido; evolución normativa; características culturales, sociales y económicas).

d) Modelo de guía de análisis: se tomó como base un trabajo elaborado por la Organización de las Naciones Unidas para la Alimentación y la Agricultura (FAO), que fue publicado en el año 2005, en donde se elaboraron una serie de aspectos conceptuales que serán aplicados para el análisis de los marcos jurídicos de los tres países seleccionados. En este caso se escogieron los siguientes aspectos: finalidad; objetivos; principios; vinculación con instrumentos internacionales y con otras normas jurídicas ambientales; y desarrollo de cuestiones ambientales y sociales, con el fin de detectar fortalezas y debilidades para cada caso.

Los datos cualitativos a recoger serán secundarios (análisis de registros escritos, tales como legislación e instrumentos internacionales). A partir del análisis comparativo de los marcos jurídicos de los tres países seleccionados, se detectaron fortalezas y debilidades para cada caso. 


\section{Antecedentes normativos}

Los bosques han sido parte importante desde el inicio de la discusión global sobre temas ambientales o de desarrollo sostenible - Conferencia de las Naciones Unidas sobre el Medio Ambiente Humano en Estocolmo en 1972-, aunque las menciones específicas a los ecosistemas forestales y su importancia para el desarrollo sostenible ocurrieron por primera vez en 1980 en el marco de la Estrategia Mundial para la Conservación, impulsada por la Unión Internacional para la Conservación de la Naturaleza, el Programa de las Naciones Unidas para el Medio Ambiente y el Fondo Mundial para la Naturaleza (Vida Silvestre) (Ministerio del Ambiente de Perú, 2016).

Cuando se hace referencia a los bosques nativos, se incluye a los ecosistemas forestales naturales compuestos predominantemente por especies arbóreas nativas maduras, con diversas especies de flora y fauna asociadas, en conjunto con el medio que las rodea (suelo, subsuelo, atmósfera, clima, recursos hídricos), conformando una trama interdependiente con características propias y múltiples funciones, que en su estado natural le otorgan al sistema una condición de equilibrio dinámico y que brinda diversos servicios ambientales a la sociedad, además de los diversos recursos naturales con posibilidad de utilización económica (Ley 26.331, artículo 2).
En relación con el marco legal antecedente, Argentina es un país que posee un sistema federal de gobierno que incluye veintitrés provincias, la Ciudad Autónoma de Buenos Aires y el Estado Nacional. Según establece la Constitución Nacional, las provincias poseen la potestad de dictar sus propias normas dentro de los límites determinados en la misma (Ferro, \& Minaverry, 2019). La Constitución Nacional, reformada en 1994, establece el derecho de todos los habitantes de gozar un ambiente sano, equilibrado y apto para el desarrollo humano. Estos derechos, incorporados en su artículo 41, se complementan con la obligación de las autoridades de garantizar la protección de los mismos, a través de la utilización racional de los recursos naturales y la preservación del patrimonio natural, cultural, y de la diversidad biológica. A su vez, en su artículo 124 establece que corresponde a las provincias el dominio originario de los recursos naturales existentes en su territorio.

A nivel internacional son varios los tratados internacionales sobre la protección de los bosques firmados por Argentina: Convenio sobre la Diversidad Biológica, Convención Marco de las Naciones Unidas sobre el Cambio Climático y Convención Internacional de Lucha contra la Desertificación en los países afectados por sequía grave o desertificación, en particular en África. Y, además, a escala internacional 
se encuentra la Declaración de Principios para el Manejo Sustentable de Bosques.

El Convenio sobre la Diversidad Biológica, que si bien no trata específicamente de los bosques, dentro de su artículo 2 se refiere a los ecosistemas terrestres, puesto que define la diversidad biológica como la variabilidad de organismos vivos de cualquier fuente, incluidos, entre otros aspectos, los ecosistemas terrestres y marinos y otros ecosistemas acuáticos y los complejos ecológicos de los que forman parte; comprende la diversidad dentro de cada especie, entre las especies y de los ecosistemas.

Al mismo tiempo que insta a tomar medidas o estrategias para la protección de los ecosistemas (terrestres y acuáticos), así como de su diversidad biológica. La Convención Marco de las Naciones Unidas sobre el Cambio Climático, en su artículo 4, establece que las partes contratantes cuentan con el compromiso de promover la gestión sostenible y promover y apoyar con su cooperación la conservación y el reforzamiento, según proceda, de los sumideros y depósitos de todos los gases de efecto invernadero no controlados por el Protocolo de Montreal, inclusive la biomasa, los bosques y los océanos, así como otros ecosistemas terrestres, costeros y marinos.

Al mismo tiempo, cabe destacar la Convención Internacional de Lucha contra la Desertificación en los países afectados por sequía grave o desertificación, en particular en África, puesto que forma parte de los compromisos por el país en materia de desarrollo sustentable.

Es el único acuerdo internacional vinculante que relaciona el medioambiente y el desarrollo con el manejo sostenible de los suelos. En sus artículos 1 y 2 establece que su objetivo es luchar contra la desertificación y mitigar los efectos de la sequía en los países afectados por sequía grave o desertificación mediante la adopción de medidas eficaces en todos los niveles. Estos son apoyados por acuerdos de cooperación y asociación internacionales en el marco de un enfoque integrado, acorde con el Programa 21, para contribuir al logro del desarrollo sostenible en las zonas afectadas. Esto implica la aplicación de estrategias integradas a largo plazo, en las zonas afectadas, que se centren simultáneamente en el aumento de la productividad de las tierras, la rehabilitación, la conservación y el aprovechamiento sostenible de los recursos de tierras e hídricos con miras a mejorar las condiciones de vida, especialmente a nivel comunitario.

Además, dentro de su artículo 1 reconoce por "degradación de las tierras" la reducción o la pérdida de la productividad biológica o económica y la complejidad de las tierras agrícolas de secano, las tierras de cultivo de regadío o las dehesas, los pastizales, los bosques y las tierras arboladas, ocasionada en zonas áridas, semiáridas 
y subhúmedas secas por los sistemas de utilización de la tierra o por un proceso o una combinación de procesos. Se incluyen las resultantes de actividades humanas y pautas de poblamiento, tales como: i) erosión del suelo ocasionada por el viento o el agua; ii) deterioro de las propiedades físicas, químicas y biológicas o de las propiedades económicas del suelo; y iii) pérdida duradera de vegetación natural.

A su vez, en su artículo 8, establece que dentro de los programas de acción nacionales una de las medidas que se debe incluir será la siguiente: Medidas para conservar los recursos naturales: i) velar por una gestión integrada y sostenible de los recursos naturales, que abarque: las tierras agrícolas y de pastoreo, la cubierta vegetal y la flora y fauna silvestres, los bosques, los recursos hídricos y su conservación, y la diversidad biológica. Y, por último, se encuentra la Declaración de Principios para el Manejo Sustentable de Bosques. Se trata de una declaración autorizada, sin fuerza jurídica obligatoria, de principios para un consenso mundial respecto de la ordenación, la conservación y el desarrollo sostenible de los bosques de todo tipo. Fue aprobada en la Conferencia de las Naciones Unidas sobre el Medio Ambiente y el Desarrollo (Cnumad) de 1992 en Río de Janeiro, donde marcó el origen del concepto de manejo forestal sostenible. Posee quince principios, pero los más relevantes para la protección de los bosques son:
- El reconocimiento de la soberanía de los Estados sobre los bosques.

- La ordenación y el desarrollo de los bosques, de conformidad con las necesidades de desarrollo y el grado de desarrollo socioeconómico, y sobre la base de una política nacional compatible con el desarrollo sostenible y la legislación.

- El reconocimiento de la función vital que cumplen los bosques de todo tipo en el mantenimiento de los procesos y el equilibrio ecológico.

- La política forestal debe reconocer y apoyar debidamente la cultura y los intereses, y respetar los derechos de las poblaciones indígenas, de sus comunidades y otras comunidades, y de los habitantes de las zonas boscosas.

- El rol fundamental de la participación social.

A nivel nacional, Argentina cuenta con la Ley General del Ambiente 25.675, sancionada y promulgada en 2002, donde establece en su artículo primero "los presupuestos mínimos para el logro de una gestión sustentable y adecuada del ambiente, la preservación y protección de la diversidad biológica y la implementación del desarrollo sustentable".

Por otro lado, en el año 2019 se sancionó la Ley de Presupuestos Mínimos de Adaptación y Mitigación al Cambio Climático Global, donde no se hace referencia específica de los bosques o sus servicios ecosis- 
témicos, pero en su artículo 24 establece como una de sus medidas y acciones de mitigación "implementar medidas que aporten a la integridad y conectividad de los ecosistemas relevantes para la captura y el almacenamiento de carbono y manejar de manera sustentable los ecosistemas intervenidos con capacidad de almacenamiento de carbono".

A nivel provincial, la Constitución de la Provincia de Buenos Aires establece en su artículo 28 que los habitantes de la Provincia tienen el derecho a gozar de un ambiente sano y el deber de conservarlo y protegerlo en su provecho y en el de las generaciones futuras. La Provincia ejerce el dominio eminente sobre el ambiente y los recursos naturales de su territorio incluyendo el subsuelo y el espacio aéreo correspondiente, el mar territorial y su lecho, la plataforma continental y los recursos naturales de la zona económica exclusiva, con el fin de asegurar una gestión ambientalmente adecuada.

Respecto a los servicios ecosistémicos, estos son definidos como los beneficios que la población obtiene de los ecosistemas (ONU, Evaluación de los Ecosistemas del Milenio, 2005).

El concepto de servicios ecosistémicos permite hacer un vínculo explícito entre el estado y funcionamiento de los ecosistemas y el bienestar humano. Esta relación puede ser directa o indirecta y los seres humanos pueden o no estar conscien- tes de su existencia (Balvanera, \& Cotler, 2007). Es decir, los bienes y servicios de los ecosistemas son, por definición, de naturaleza inherentemente pública (Howarth, \& Wilson, 2002), y según Daily (1997), citado por Howarth y Wilson (2002, p. 432), contienen todas las condiciones y procesos a través de los cuales los ecosistemas naturales y las especies que los componen, sostienen y satisfacen la vida humana. Definido así, los bienes y servicios del ecosistema brindan beneficios a la sociedad en su conjunto, además de los beneficios que proporcionan a los individuos (Howarth, \& Wilson, 2002).

En relación con la conservación o protección de estos servicios aún no ha sido regulada autónomamente en la normativa nacional ambiental (Minaverry, 2017).

Sin embargo, en cuanto a su protección jurídica internacional se destacan los diecisiete Objetivos de Desarrollo Sostenible de las Naciones Unidas, elaborados a fines del año 2015 y que se proyectan hacia 2030.

El n. ${ }^{\circ} 15$, en particular, establece: "Promover el uso sostenible de los ecosistemas terrestres, luchar contra la desertificación, detener e invertir la degradación de las tierras y frenar la pérdida de la biodiversidad biológica”. Entre sus metas, se destaca la siguiente:

15.1: Para 2020, velar por la conservación, el restablecimiento y el uso sostenible de 
los ecosistemas terrestres y los ecosistemas interiores de agua dulce y los servicios que proporcionan, en particular los bosques, los humedales, las montañas y las zonas áridas, en consonancia con las obligaciones contraídas en virtud de acuerdos internacionales (Minaverry, 2020).

\section{Marco conceptual}

Los bosques son los ecosistemas terrestres más extensos, los cuales ocupan el 30 $\%$ de la superficie emergida del planeta. Además, poseen un gran valor en términos de biodiversidad. Se estima que estos ecosistemas forestales albergan, al menos, el $75 \%$ de las especies continentales y una parte importante de la biomasa terrestre, al mismo tiempo que son hábitat y fuente de subsistencia de cientos de millones de personas (entre las cuales se destacan las comunidades indígenas), especialmente en los países menos desarrollados. A su vez, desempeñan funciones ambientales de gran importancia a distintas escalas, desde la local hasta la global (Ruiz Pérez, García Fernández, \& Sayer, 2007).

Estos ecosistemas tienen un rol fundamental en la regulación climática, el mantenimiento de las fuentes y caudales de agua, y la conservación de los suelos, a la vez que proveen bienes indispensables, como alimentos, maderas y medicinas. Es decir, los bosques forman un sistema natural complejo, que, junto a los mares y océanos, constituyen el sustento esencial para la vida en la tierra. Son indispensables para el mantenimiento de la biodiversidad de los ecosistemas y para la regulación del clima del planeta (Secretaría de Ambiente y Desarrollo Sustentable, 2005).

Desde el punto de vista de la conservación de la biodiversidad, debe destacarse, como ya se mencionó anteriormente, que el bosque es hábitat de una gran variedad de flora y fauna silvestres, y que su destrucción implicaría el riesgo de extinción de numerosas especies. El manejo de los bosques repercute inevitablemente en la conservación de los demás recursos naturales renovables, en la diversidad biológica y en el equilibrio ecológico local, regional y global (Secretaría de Ambiente y Desarrollo Sustentable, 2005).

Existen dos tipos de bosques: los nativos y los implantados. Por un lado, los implantados son aquellos instalados por el hombre mediante la plantación de especies nativas o exóticas; que surgen como una necesidad, debido a la tala indiscriminada de los bosques nativos, es decir, como una necesidad de producir recursos forestales con fines económicos, buscando reducir el impacto ambiental que genera la deforestación. Estos bosques se concentran en la región de la Mesopotamia (misiones, corrientes y entre ríos), delta del río Paraná, Buenos Aires, Córdoba y Neuquén (Senasa, 2014). 
Por otro lado, los bosques nativos son aquellos ecosistemas forestales naturales que no han sido modificados por el hombre, en los que predominan las especies arbóreas nativas con una gran diversidad de flora y fauna. Están compuestos por más de noventa especies, entre las que se encuentran: quebrachos colorados y blancos, algarrobos, lapachos, inciensos, cedros, guatambúes, jacarandas, peteribís, palos santos, alerces, ñandubays y sauces (Senasa, 2014).

La Ley Nacional n. 26.331 (sancionada en el año 2007) sobre Presupuestos Mínimos de Protección Ambiental de los Bosques Nativos establece, en su artículo 5, los principales servicios ambientales que los bosques nativos brindan a la sociedad:

- Regulación hídrica.

- Conservación de la biodiversidad.

- Conservación del suelo y de la calidad del agua.

- Fijación de emisiones de gases con efecto invernadero.

- Contribución a la diversificación y belleza del paisaje.

- Defensa de la identidad cultural.

A principios de siglo, los bosques naturales cubrían un tercio del territorio argentino y representaban más de 100 millones de hectáreas. Actualmente, en Argentina los bosques nativos ocupan una superficie de 47.9 millones de hectáreas, aproxima- damente, distribuidas en siete regiones forestales: Parque Chaqueño; Yungas; Selva Paranaense; Monte; Espinal; Bosque Andino Patagónico; y Delta e islas del río Paraná (Ministerio de Ambiente y Desarrollo Sostenible, 2021); y 1.2 millones de hectáreas, aproximadamente, de monte implantado (Senasa, 2014).

Concretamente, en cuanto a la distribución de los bosques nativos a lo largo de las regiones forestales, el $67 \%$ se encuentra en la región Parque Chaqueño, seguida por los bosques de la región Espinal, que representan el $12 \%$. Sin embargo, dentro de cada región forestal, las selvas de las Yungas (68 \%) y la Selva Paranaense (61 \%) son las que tienen más superficie ocupada por bosques nativos (Ministerio de Ambiente y Desarrollo Sostenible, 2021).

$\mathrm{Al}$ mismo tiempo, los bosques cumplen una importante función que consiste en actuar como reservorios de dióxido de carbono (uno de los principales gases que ocasionan el efecto invernadero), al punto tal que cerca de un $20 \%$ de las emisiones globales de estos gases provienen de la deforestación y la degradación de los bosques. Y, en forma indirecta, contribuyen a la conservación de las fuentes de agua, la prevención de inundaciones y la protección para los suelos (Fundación Vida Silvestre, s.f.).

Por otra parte, la situación de los bosques en Argentina es crítica, debido a que los bosques nativos están en proceso de 
degradación por la deforestación. Desde 1990 en Argentina se perdieron $7.6 \mathrm{mi}$ llones de hectáreas de bosques, que en la última década se ha llevado a razón de 300000 hectáreas anuales. Un bosque degradado es el que pierde su diversidad biológica, las posibilidades de sostener actividades económicas y que ese ambiente provea servicios ambientales, como la regulación del clima y del agua. Actualmente, se conserva el $27 \%$ de la superficie originalmente ocupada por bosques nativos, lo que significa que Argentina está en una verdadera emergencia forestal (Fundación Vida Silvestre, s.f.).

La Ley de Bosques no impide más desmontes, sino que establece zonas rojas, donde la tala está prohibida; zonas amarillas, donde también está prohibida la tala y se autoriza un uso sustentable del recurso; y zonas verdes, donde el cambio de uso de la tierra puede autorizarse a avanzar sobre los bosques. Sin embargo, una de las coartadas llevadas a cabo por las provincias para no cumplir con la ley ha sido el cambio de la zonificación para autorizar la expansión de emprendimientos agrícolas y ganaderos.

Ante este panorama, respecto a la degradación de los bosques, surge de manera evidente la necesidad de elaborar e implementar políticas de Estado tendientes a conservar y restaurar los bosques nativos para el presente y las generaciones futuras, tal cual lo establece el mandato constitucional. Ahora bien, la ejecución de estas políticas, cuyos alcances se deberán extender en el tiempo y trascender los gobiernos, requiere, por una parte, la decisión del gobierno y de la sociedad en su conjunto para llevarlas a cabo, y por la otra, la disponibilidad de las herramientas y los insumos técnicos específicos para diseñarlas (Secretaría de Ambiente y Desarrollo Sustentable, 2005).

Resulta crucial la protección de estos ecosistemas forestales no solo para proteger las especies de flora y fauna que habitan en ellos, sino también para prevenir las inundaciones, la erosión, los incendios; evitar el avance de la desertificación; asegurar la calidad del agua que consumimos y del aire que respiramos; garantizar a las generaciones presentes y futuras aquellos bienes y servicios que ofrecen (como la madera, leña, frutos, miel, alimentos, medicamentos, turismo, recreación); y para preservar la identidad cultural de los pueblos que viven en y de los bosques (Ministerio de Ambiente y Desarrollo Sostenible, s.f.).

En cuanto a los servicios ecosistémicos, estos cumplen la función básica de satisfacer las necesidades humanas y generar su bienestar, incidiendo directamente sobre la calidad de vida. La producción de alimentos es uno de los principales servicios que prestan los ecosistemas, ya que posibilitan a toda la población el acceso a ellos, a partir de los cuales satisfagan las necesi- 
dades diarias alimentarias que les permita llevar una vida sana y activa. A la vez que prestan otros servicios esenciales, tales como la provisión de agua, la regulación climática, recreativos, de preservación de valores culturales, entre otros.

El concepto de servicios ofrecidos por los ecosistemas hacia las poblaciones humanas surge a consecuencia del movimiento ambientalista de finales de los años sesenta. En esta época, se hace patente la crisis ambiental y se inician cuestionamientos acerca de los impactos severos en la capacidad del planeta para mantenerse y producir suficientes bienes para ser consumidos por las poblaciones humanas. Junto con una lista de los problemas ambientales más severos surge la primera relación de servicios ecosistémicos que se proveen a las sociedades en un esfuerzo por comunicar a los tomadores de decisiones y al público en general acerca del estrecho vínculo entre el bienestar humano y el mantenimiento de las funciones básicas del planeta. Es decir, el concepto de servicios ecosistémicos surge como consecuencia de la necesidad de enfatizar esta estrecha relación que existe entre los ecosistemas y el bienestar de las poblaciones humanas (Balvanera, \& Cotler, 2007).

Los servicios ecosistémicos se definen como los componentes y procesos de los ecosistemas que son consumidos, disfrutados o que conducen a aumentar el bienestar humano, tomando en cuenta la demanda de los beneficiarios, así como la dinámica de los ecosistemas (Balvanera $e t$ al., s.f.). Pero, a su vez, existen varias definiciones más sobre estos servicios, citadas por Camacho Valdez y Ruiz Luna (2012), que se presentan a continuación en orden cronológico:

- Las condiciones y procesos a través de los cuales los ecosistemas naturales, y las especies que los constituyen, sustentan y satisfacen la vida humana (Daily, 1997).

- Los bienes (como alimentos) y servicios (como asimilación de residuos) de los ecosistemas, que representan los beneficios que la población humana obtiene, directa o indirectamente, de las funciones de los ecosistemas (Costanza et al., 1997).

- Funciones del ecosistema: capacidad de los procesos y componentes naturales para proporcionar bienes y servicios que satisfacen las necesidades humanas, directa o indirectamente (De Groot et al., 2002).

- Los beneficios que la población obtiene de los ecosistemas ( $\mathrm{MA}^{3}{ }^{3} 2003$ ).

- Aquellas funciones o procesos ecológicos que directa o indirectamente contribuyen al bienestar humano o tienen un potencial para hacerlo en el futuro (US EPA, 2004).

3 Evaluación de los Ecosistemas del Milenio. 
- Son componentes de la naturaleza disfrutados, consumidos o directamente usados para producir bienestar humano (Boyd, \& Banzhaf, 2007).

- Son los aspectos de los ecosistemas utilizados (activa o pasivamente) para producir bienestar humano (Fisher et al., 2009).

Como vimos anteriormente, existen múltiples definiciones respecto de los servicios ecosistémicos realizadas por varios autores; sin embargo, todas coinciden en que cuando se hace mención de estos, se refieren a los beneficios que las poblaciones humanas obtienen de los ecosistemas para la satisfacción de sus necesidades. Estos servicios incluyen: ${ }^{4}$

- Los bienes o recursos naturales, como el agua o los alimentos.

- Los procesos ecosistémicos que regulan las condiciones en las que los seres humanos habitan, como la regulación del clima o de la erosión.

- La contribución de los ecosistemas a experiencias que benefician directa o indirectamente a las sociedades, como el sentido de pertenencia o la recreación.

$4 \quad$ Balvanera, P. et al. (s.f.). Marcos conceptuales interdisciplinarios para el estudio de los servicios ecosistémicos en América Latina. En: P. Laterra, E. G. Jobbágy, \& J. M. Paruelo (Eds.), Valoración de servicios ecosistémicos. Conceptos, herramientas y aplicaciones para el ordenamiento territorial (pp. 38-67). Universidad Nacional Autónoma de México.
- Los procesos ecológicos básicos que permiten que se provean los anteriores.

Por otro lado, existe una clasificación realizada por diferentes autores de veintitrés funciones básicas de los ecosistemas agrupadas en cuatro categorías principales, de las cuales se derivan diferentes bienes y servicios:

- Funciones de regulación: relacionadas con la capacidad de los ecosistemas para regular procesos ecológicos esenciales y sostener sistemas vitales, a través de ciclos biogeoquímicos y otros procesos biológicos. Estas funciones proporcionan muchos servicios que tienen beneficios directos e indirectos para las poblaciones humanas, como el mantenimiento de aire limpio, depuración del agua, prevención de inundaciones y mantenimiento de tierra cultivable, entre otros.

- Funciones de hábitat: los ecosistemas naturales proporcionan hábitat de refugio y reproducción para plantas y animales, contribuyendo a la conservación biológica y la diversidad genética. Estas funciones proporcionan servicios, como el mantenimiento de la diversidad biológica y genética, y de especies comercialmente aprovechables.

- Funciones de producción: son los procesos fotosintéticos y autótrofos en general, a partir de los cuales los organismos 
autoabastecen sus requerimientos orgánicos por medio de compuestos inorgánicos y que también son sustento de consumidores de distinto orden, para generar una mayor variedad de biomasa. Esta variedad de estructuras proporciona bienes y servicios para el consumo humano, que van desde alimentos y materias primas hasta recursos energéticos y medicinales.

- Funciones de información: los ecosistemas proporcionan funciones de referencia y contribuyen al mantenimiento de la salud humana, proporcionando oportunidades de enriquecimiento espiritual, desarrollo cognitivo, recreación y experiencias estéticas (paisaje). ${ }^{5}$

Otra aproximación para clasificar los servicios ecosistémicos es la derivada de la Evaluación de los Ecosistemas del Milenio (MA, 2005), que es probablemente la más difundida y aceptada. Ofrece un sistema de clasificación con propósitos puramente operacionales basado en cuatro líneas funcionales dentro del marco conceptual de MA, que incluyen servicios de soporte, aprovisionamiento, regulación y culturales con la intención de facilitar la toma de decisiones. Esas cuatro líneas funcionales son las siguientes:

5 Camacho Valdez, V., \& Ruiz Luna, A. (2012). Marco conceptual y clasificación de los servicios ecosistémicos. Rev. Biociencias, 1(4), 3-15. http://revistabiociencias.uan.mx/index.php/BIOCIENCIAS/article/ view/19
- Servicios de soporte: son necesarios para la producción de todos los demás servicios ecosistémicos (formación de suelos, reciclaje de nutrientes, producción primaria).

- Aprovisionamiento: son productos obtenidos del ecosistema (alimentos, agua dulce, fibras, leña, bioquímicos, recursos genéticos).

- Regulación: son beneficios obtenidos de la regulación de los procesos del ecosistema (regulación del clima, regulación de enfermedades, regulación y saneamiento del agua, polinización).

- Culturales: son beneficios no-materiales que la gente obtiene de los ecosistemas (espirituales y religiosos, recreativos y turísticos, estéticos, inspirativos, educativos, identidad de sitio, herencia cultu$\mathrm{ral}){ }^{6}$

En síntesis, el concepto de servicios ecosistémicos o servicios ambientales permite hacer un vínculo explícito entre el estado y funcionamiento de los ecosistemas y el bienestar humano. Esta relación puede ser directa o indirecta y los seres humanos pueden o no estar conscientes de su existencia (Balvanera, \& Cotler, 2007).

6 Camacho Valdez, V., \& Ruiz Luna, A. (2012). Marco conceptual y clasificación de los servicios ecosistémicos. Rev. Biociencias, 1(4), 3-15. http://revistabiociencias.uan.mx/index.php/BIOCIENCIAS/article/ view/19 
Normativa relacionada con los bosques y sus servicios ecosistémicos: los casos de Argentina, Colombia y Perú

Dentro de esta sección, se enuncian cada una de las leyes nacionales y decretos que poseen Argentina, Colombia y Perú, que se relacionan directamente con la temática de los bosques nativos y sus servicios ecosistémicos.

Las leyes nacionales fueron comparadas y caracterizadas en formato de cuadro, tomando como base el trabajo elaborado por la Organización de las Naciones Uni- das para la Alimentación y la Agricultura (FAO), que fue publicado en el año 2005 , en donde se elaboraron una serie de aspectos conceptuales que fueron aplicados para el análisis de los marcos jurídicos de los tres países seleccionados. En este caso, se seleccionaron los siguientes aspectos: finalidad; objetivos; principios; vinculación con instrumentos internacionales y con otras normas jurídicas ambientales; y desarrollo de cuestiones ambientales y sociales, que a continuación se presentan (tablas 1-6).

Tabla 1. Finalidad de cada ley

\begin{tabular}{|c|c|c|c|}
\hline & Argentina & Colombia & Perú \\
\hline \multirow[t]{2}{*}{ Finalidad } & $\begin{array}{l}\text { Ley 13.273: } \\
\text { La defensa, mejoramiento y } \\
\text { ampliación de los bosques. }\end{array}$ & \multirow{2}{*}{$\begin{array}{c}\text { Ley 1.753: } \\
\text { Dictar/expedir el } \\
\text { Plan Nacional de } \\
\text { Desarrollo 2014- } \\
2018 \text { “Todos por un } \\
\text { nuevo país”. }\end{array}$} & $\begin{array}{c}\text { Ley 30.215: } \\
\text { Asegurar la permanencia de los } \\
\text { beneficios generados por los ecosis- } \\
\text { temas. }\end{array}$ \\
\hline & $\begin{array}{l}\text { Ley 26.331: } \\
\text { El enriquecimiento, restaura- } \\
\text { ción, conservación, aprovecha- } \\
\text { miento y manejo sostenible de } \\
\text { los bosques nativos, y de los } \\
\text { servicios ambientales que es- } \\
\text { tos brindan a la sociedad. }\end{array}$ & & $\begin{array}{l}\text { Ley 29.763: } \\
\text { Promover la conservación, la pro- } \\
\text { tección, el incremento y el uso soste- } \\
\text { nible del patrimonio forestal y de la } \\
\text { fauna silvestre dentro del territorio } \\
\text { nacional. }\end{array}$ \\
\hline
\end{tabular}

Fuente: elaboración propia (2021). 
Tabla 2. Objetivos planteados por cada ley

\begin{tabular}{|c|c|c|c|}
\hline & Argentina & Colombia & Perú \\
\hline \multirow[t]{2}{*}{ Objetivos } & $\begin{array}{c}\text { Ley 13.273: } \\
\text { No se hace mención explícita de } \\
\text { los objetivos. }\end{array}$ & \multirow[t]{2}{*}{$\begin{array}{c}\text { Ley 1.753: } \\
\text { Construir una Colombia } \\
\text { en paz, equitativa y edu- } \\
\text { cada, en armonía con los } \\
\text { propósitos del gobierno } \\
\text { nacional, con las mejores } \\
\text { prácticas y estándares } \\
\text { internacionales y con la } \\
\text { visión de planificación de } \\
\text { largo plazo prevista por } \\
\text { los Objetivos de Desarrollo } \\
\text { Sostenible. }\end{array}$} & $\begin{array}{c}\text { Ley 30.215: } \\
\text { Promueve, regula y supervisa } \\
\text { los mecanismos de retribución } \\
\text { por servicios ecosistémicos que } \\
\text { se derivan de acuerdos volun- } \\
\text { tarios que establecen acciones } \\
\text { de conservación, recuperación } \\
\text { y uso sostenible para asegurar } \\
\text { la permanencia de los ecosis- } \\
\text { temas. }\end{array}$ \\
\hline & $\begin{array}{c}\text { Ley 26.331: } \\
\text { • Promover la conservación me- } \\
\text { diante el Ordenamiento Territorial } \\
\text { de los Bosques Nativos y la regula- } \\
\text { ción de la expansión de la frontera } \\
\text { agropecuaria y de cualquier otro } \\
\text { cambio de uso del suelo. } \\
\text { - Implementar las medidas nece- } \\
\text { sarias para regular y controlar } \\
\text { la disminución de la superficie } \\
\text { de bosques nativos existentes, } \\
\text { tendiendo a lograr una superficie } \\
\text { perdurable en el tiempo. } \\
\text { - Mejorar y mantener los procesos } \\
\text { ecológicos y culturales en los bos- } \\
\text { ques nativos que beneficien a la } \\
\text { sociedad. } \\
\text { - Hacer prevalecer los principios } \\
\text { precautorio y preventivo man- } \\
\text { teniendo bosques nativos, cuyos } \\
\text { beneficios ambientales o los daños } \\
\text { ambientales que su ausencia gene- } \\
\text { rase aún no puedan demostrarse } \\
\text { con las técnicas disponibles en la } \\
\text { actualidad. } \\
\text { • Fomentar las actividades de en- } \\
\text { riquecimiento, conservación, res- } \\
\text { tauración, mejoramiento y manejo } \\
\text { sostenible de los bosques nativos. }\end{array}$ & & $\begin{array}{l}\text { Ley 29.763: } \\
\text { Establecer el marco legal para } \\
\text { regular, promover y supervisar } \\
\text { la actividad forestal y de la } \\
\text { fauna silvestre para lograr su } \\
\text { finalidad. }\end{array}$ \\
\hline
\end{tabular}

Fuente: elaboración propia (2021). 
Tabla 3. Principios en los que se basan cada una de las leyes

\begin{tabular}{|c|c|c|c|}
\hline & Argentina & Colombia & Perú \\
\hline \multirow[t]{3}{*}{ Principios } & Ley 13.273: & Ley 1.753: & Ley 30.215: \\
\hline & $\begin{array}{l}\text { No se plantean los } \\
\text { principios a los que } \\
\text { estaría sujeta. }\end{array}$ & \multirow{2}{*}{$\begin{array}{l}\text { No se hace } \\
\text { mención explíci- } \\
\text { ta de los prin- } \\
\text { cipios a los que } \\
\text { estaría sujeta. }\end{array}$} & $\begin{array}{c}\text { No se hace mención explícita de } \\
\text { los principios a los que estaría su- } \\
\text { jeta. }\end{array}$ \\
\hline & $\begin{array}{c}\text { Ley 26.331: } \\
\text { Dentro de los objeti- } \\
\text { vos, se enuncian los } \\
\text { principios precautorio } \\
\text { y preventivo. }\end{array}$ & & $\begin{array}{l}\text { Ley 29.763: } \\
\text { Principios de gobernanza forestal y } \\
\text { de la fauna silvestre; participación } \\
\text { en la gestión forestal; de consulta } \\
\text { previa libre e informada; de equi- } \\
\text { dad e inclusión social; de intercul- } \\
\text { turalidad, conocimientos tradicio- } \\
\text { nales y cosmovisión; de enfoque } \\
\text { ecosistémico; de sostenibilidad de } \\
\text { la gestión del patrimonio forestal y } \\
\text { de la fauna silvestre de la Nación; } \\
\text { de dominio eminencial del Estado; } \\
\text { de valoración integral; de origen } \\
\text { legal; de eficiencia y mejoramiento } \\
\text { continuo; de integración con otros } \\
\text { marcos normativos; y de transpar- } \\
\text { encia y rendición de cuentas. }\end{array}$ \\
\hline
\end{tabular}

Fuente: elaboración propia (2021). 
Tabla 4. Mención de instrumentos internacionales y normas ambientales que se relacionan con las presentes leyes de este trabajo y que se enuncian dentro de sus respectivos textos

\begin{tabular}{|c|c|c|c|}
\hline & Argentina & Colombia & Perú \\
\hline \multirow[t]{2}{*}{$\begin{array}{l}\text { Vinculación con instrumentos } \\
\text { internacionales y con otras } \\
\text { normas jurídicas ambientales }\end{array}$} & $\begin{array}{l}\text { Ley 13.273: } \\
\text { Se hace mención de las } \\
\text { leyes nacionales } 19.995 \\
\text { y 21.990, las cuales tien- } \\
\text { den a una complemen- } \\
\text { tación y actualización } \\
\text { de las disposiciones } \\
\text { contenidas en esta ley. }\end{array}$ & \multirow[t]{2}{*}{$\begin{array}{c}\text { Ley 1.753: } \\
\text { Convención ram- } \\
\text { sar y Convención } \\
\text { Internacional } \\
\text { sobre el Comer- } \\
\text { cio de Especies } \\
\text { Amenazadas de } \\
\text { Fauna y Flora } \\
\text { Silvestres (Cites). }\end{array}$} & $\begin{array}{l}\text { Ley 30.215: } \\
\text { Se hace mención de la Ley } 27867 \text { (Ley } \\
\text { Orgánica de Gobiernos Regionales); la } \\
\text { Ley } 27972 \text { (Ley Orgánica de Municipa- } \\
\text { lidades); la Ley } 29785 \text { (Ley del Derecho } \\
\text { a la Consulta Previa a los Pueblos } \\
\text { Indígenas u Originarios), reconocida } \\
\text { en el Convenio 169 de la Organización } \\
\text { Internacional del Trabajo (oit), y su } \\
\text { reglamento, el Decreto Supremo 001- } \\
\text { 2012-mc. }\end{array}$ \\
\hline & $\begin{array}{c}\text { Ley 26.331: } \\
\text { El Convenio 169 de la } \\
\text { Organización Interna- } \\
\text { cional del Trabajo (oit); } \\
\text { la Ley } 26.160 \text { y la Ley } \\
\text { 24.071, referidas a las } \\
\text { Comunidades Indígenas } \\
\text { y Campesinas. Y, ade- } \\
\text { más, a lo largo de sus ar- } \\
\text { tículos, se hace referen- } \\
\text { cia a la Ley 25.675; la Ley } \\
\text { 25.831 (Régimen de Libre } \\
\text { Acceso a la Información } \\
\text { Pública Ambiental); y la } \\
\text { Ley 24.156. }\end{array}$ & & $\begin{array}{c}\text { Ley 29.763: } \\
\text { El Convenio } 169 \text { de la Organización } \\
\text { Internacional del Trabajo (oit); y la } \\
\text { Declaración de la Organización de las } \\
\text { Naciones Unidas sobre los Derechos } \\
\text { de los Pueblos Indígenas. }\end{array}$ \\
\hline
\end{tabular}

Fuente: elaboración propia (2021). 
Tabla 5. Qué plantea cada ley respecto a lo ambiental

\begin{tabular}{|c|c|c|c|}
\hline & Argentina & Colombia & Perú \\
\hline \multirow[t]{2}{*}{$\begin{array}{c}\text { Componente am- } \\
\text { biental }\end{array}$} & $\begin{array}{l}\text { Ley 13.273: } \\
\text { Esta ley se basa en todo lo } \\
\text { relativo a bosques y tierras } \\
\text { forestales. }\end{array}$ & \multirow{2}{*}{$\begin{array}{l}\text { Ley 1.753: } \\
\text { Establece que debe formular- } \\
\text { se una política de crecimiento } \\
\text { verde de largo plazo, en la } \\
\text { cual se definan los objetivos y } \\
\text { metas de crecimiento econó- } \\
\text { mico sostenible. Por otra par- } \\
\text { te, plantea que se debe llevar } \\
\text { a cabo el seguimiento de los } \\
\text { planes de adaptación y miti- } \\
\text { gación del cambio climático. } \\
\text { Además, el Ministerio de Am- } \\
\text { biente y Desarrollo Sostenible } \\
\text { deberá elaborar una política } \\
\text { nacional de lucha contra la } \\
\text { deforestación, que contendrá } \\
\text { un plan de acción dirigido a } \\
\text { evitar la pérdida de bosques } \\
\text { naturales para el año } 2030 . \\
\text { Y, a su vez, establecerá un } \\
\text { programa de monitoreo de } \\
\text { los humedales que evalúe el } \\
\text { estado de conservación de los } \\
\text { mismos. }\end{array}$} & $\begin{array}{l}\text { Ley 30.215: } \\
\text { Hace referencia a los meca- } \\
\text { nismos de retribución por } \\
\text { servicios ecosistémicos. }\end{array}$ \\
\hline & $\begin{array}{l}\text { Ley 26.331: } \\
\text { Esta Ley establece los presu- } \\
\text { puestos mínimos de protec- } \\
\text { ción ambiental para el enri- } \\
\text { quecimiento, restauración, } \\
\text { conservación, aprovecha- } \\
\text { miento y manejo sostenible } \\
\text { de los bosques nativos, y de } \\
\text { los servicios ambientales } \\
\text { que estos brindan a la socie- } \\
\text { dad. }\end{array}$ & & $\begin{array}{l}\text { Ley 29.763: } \\
\text { Plantea la planificación fores- } \\
\text { tal y de la fauna silvestre, al } \\
\text { mismo tiempo que propone la } \\
\text { zonificación forestal. }\end{array}$ \\
\hline
\end{tabular}

Fuente: elaboración propia (2021). 
Tabla 6. Qué plantea cada ley en torno al ámbito social

\begin{tabular}{|c|c|c|c|}
\hline & Argentina & Colombia & Perú \\
\hline \multirow[t]{2}{*}{ Componente social } & $\begin{array}{c}\text { Ley 13.273: } \\
\text { No se hace mención } \\
\text { específica de la parti- } \\
\text { cipación social; pero sí } \\
\text { de ciertas condiciones } \\
\text { y aprovechamientos } \\
\text { de los bosques y tie- } \\
\text { rras forestales para las } \\
\text { personas. }\end{array}$ & \multirow{2}{*}{$\begin{array}{c}\text { Ley 1.753: } \\
\text { Este Plan Nacional } \\
\text { de Desarrollo se } \\
\text { basa en tres pilares: } \\
\text { la paz (construir } \\
\text { una paz sostenible } \\
\text { bajo un enfoque de } \\
\text { goce efectivo de de- } \\
\text { rechos); la equidad } \\
\text { (una sociedad con } \\
\text { oportunidades para } \\
\text { todos); y la educa- } \\
\text { ción (donde esta se } \\
\text { asume como el más } \\
\text { poderoso instru- } \\
\text { mento de igualdad } \\
\text { social y crecimiento } \\
\text { económico a largo } \\
\text { plazo). }\end{array}$} & $\begin{array}{l}\text { Ley 30.215: } \\
\text { Esta Ley se aplica directamente a las personas } \\
\text { naturales o jurídicas, públicas o privadas, que } \\
\text { implementen mecanismos de retribución por } \\
\text { servicios ecosistémicos. A su vez, se promueve } \\
\text { la creación de una plataforma conformada } \\
\text { por diferentes actores mediante la cual se } \\
\text { monitoree el cumplimiento de los acuerdos y } \\
\text { se supervise la transparencia en la retribución } \\
\text { bajo la estrategia de financiamiento que se } \\
\text { considere adecuada. }\end{array}$ \\
\hline & $\begin{array}{l}\text { Ley 26.331: } \\
\text { Dentro de esta Ley, se } \\
\text { establece que se debe } \\
\text { realizar el Ordena- } \\
\text { miento de los Bosques } \\
\text { Nativos existentes en } \\
\text { su territorio mediante } \\
\text { un proceso participa- } \\
\text { tivo. Además, plantea } \\
\text { que se debe garantizar } \\
\text { el acceso a la infor- } \\
\text { mación de los pueblos } \\
\text { indígenas, originarios, } \\
\text { de las comunidades } \\
\text { campesinas y otras } \\
\text { relacionadas. }\end{array}$ & & $\begin{array}{l}\text { Ley 29.763: } \\
\text { Uno de los principios en los que se basa es el } \\
\text { de participación en la gestión forestal, el cual } \\
\text { otorga a toda persona el derecho y el deber de } \\
\text { participar responsablemente en los procesos } \\
\text { para la toma de decisiones respecto de las po- } \\
\text { líticas, gestión y medidas relativas a los ecosis- } \\
\text { temas forestales y otros de vegetación silvestre } \\
\text { y sus componentes. Además, se encuentra la } \\
\text { Comisión Nacional Forestal y de Fauna Silves- } \\
\text { tre (Conafor), que es la entidad consultiva del } \\
\text { Servicio Nacional Forestal y de Fauna Silvestre } \\
\text { (Serfor) de alto nivel en materia de participa- } \\
\text { ción, consulta e intercambio de información } \\
\text { sobre la política nacional forestal y de la fauna } \\
\text { silvestre. }\end{array}$ \\
\hline
\end{tabular}

Fuente: elaboración propia (2021).

Al mismo tiempo, se incorporó un nue- se trata de una elaboración propia), donde vo aspecto dentro de la caracterización de se explica cómo se relaciona directamente cada ley (que no pertenece al documento cada ley con los bosques y sus servicios ecode la FAO [2005], sino que, por el contrario, sistémicos (tabla 7). 


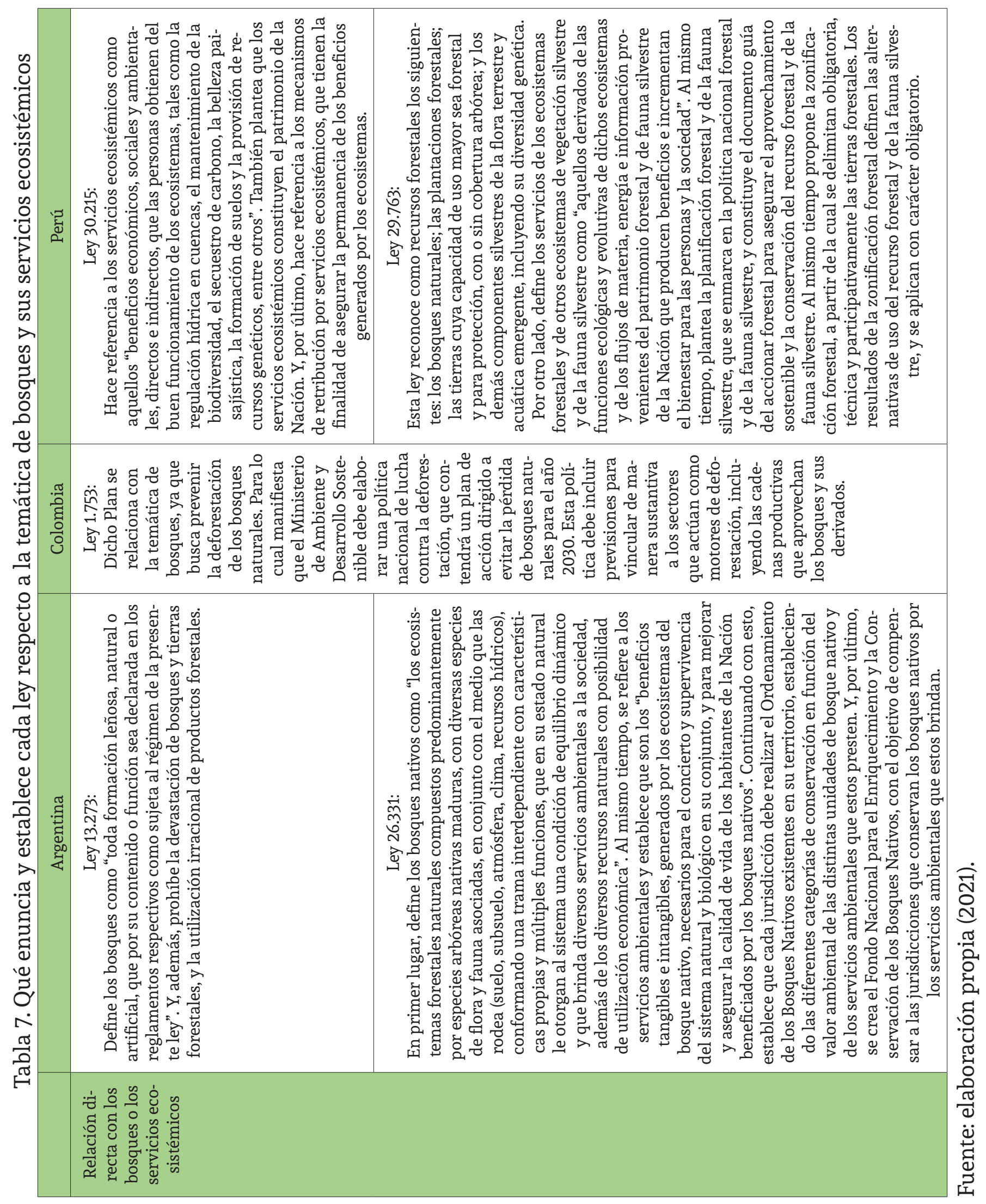


Prosiguiendo con la mención y caracterización de la normativa relacionada con los bosques y sus servicios ecosistémicos, a continuación se enuncian los decretos con los que cuenta cada país. En los casos de Perú y Colombia, además de las leyes nacionales que se enunciaron anteriormente, cuentan con decretos que regulan también la temática de este trabajo.

En primer lugar, el caso de Perú posee el Decreto Legislativo n.o 1220, el cual establece medidas para la lucha contra la tala ilegal. Para lo cual su objeto consiste en declarar de necesidad pública, interés nacional y de ejecución prioritaria las acciones de prohibición contra la tala ilegal y el tráfico ilegal de productos forestales maderables, así como las actividades relacionadas con estos.

En segundo lugar, el caso de Colombia cuenta con el Decreto Ley 870 mediante el cual se establece el pago por servicios ambientales y otros incentivos a la conservación. Busca establecer las directrices para el desarrollo de estos pagos, que permitan el mantenimiento y generación de servicios ambientales en áreas y ecosistemas estratégicos, a través de acciones de preservación y restauración.

Concretamente, el pago por servicios ambientales es el incentivo económico en dinero o en especie que reconocen los interesados de los servicios ambientales a los propietarios, poseedores u ocupantes de buena fe, exenta de culpa por las ac- ciones de preservación y restauración en áreas y ecosistemas estratégicos mediante la celebración de acuerdos voluntarios entre los interesados y beneficiarios de los servicios ambientales.

Finalmente, Colombia, además, posee el Decreto 1.791, el cual regula las actividades de la administración pública y de los particulares respecto al uso, manejo, aprovechamiento y conservación de los bosques y la flora silvestre con el fin de lograr un desarrollo sostenible. Esta norma se guía por algunos principios generales que se relacionan con los bosques:

- Los bosques, en tanto parte integrante y soporte de la diversidad biológica, étnica y de la oferta ambiental, son un recurso estratégico de la Nación y, por lo tanto, su conocimiento y manejo son tarea esencial del Estado con apoyo de la sociedad civil. Por su carácter de recurso estratégico, su utilización y manejo debe enmarcarse dentro de los principios de sostenibilidad consagrados por la Constitución Política como base del desarrollo nacional.

- Las acciones para el desarrollo sostenible de los bosques son una tarea conjunta y coordinada entre el Estado, la comunidad y el sector privado, quienes propenderán para que se optimicen los beneficios de los servicios ambientales, sociales y económicos de los bosques. 
- El aprovechamiento sostenible de la flora silvestre y de los bosques es una estrategia de conservación y manejo del recurso. Por lo tanto, el Estado debe crear un ambiente propicio para las inversiones en materia ambiental y para el desarrollo del sector forestal.

- Gran parte de las áreas boscosas naturales del país, se encuentran habitadas. Por lo tanto, se apoyará la satisfacción de las necesidades vitales, la conservación de sus valores tradicionales y el ejercicio de los derechos de sus moradores dentro de los límites del bien común.

Finalmente, a partir de todo el marco normativo presentado, se puede observar que los tres países buscan la conservación y protección de los bosques. No obstante, en los tres casos se observa una profusa normativa fragmentada que regula solo aspectos parciales de los bosques.

\section{Consideraciones finales}

A modo de conclusión, se destaca que los bosques nativos poseen un gran valor para la biodiversidad y la vida humana, gracias a todos los servicios/beneficios que brindan. Estos ecosistemas no solo albergan la mayor parte de la biodiversidad global, sino que también son hábitat y fuente de subsistencia de millones de personas (entre las cuales se destacan las comunidades indígenas). Y, a su vez, desempeñan fun- ciones ambientales de gran importancia a distintas escalas, desde la local hasta la global.

Los bosques nativos tienen un rol fundamental en la regulación climática, el mantenimiento de las fuentes y caudales de agua, y la conservación de los suelos, a la vez que proveen bienes indispensables, como alimentos, maderas y medicinas a la sociedad. A su vez, proveen servicios ecosistémicos (de soporte, de aprovisionamiento, de regulación y culturales) que cumplen la función básica de satisfacer las necesidades humanas y generar su bienestar, incidiendo directamente sobre la calidad de vida.

Por lo que el manejo de los bosques repercute inevitablemente en la conservación de los demás recursos naturales renovables, en la diversidad biológica y en el equilibrio ecológico local, regional y global. No obstante, la situación de los bosques en Argentina es crítica, ya que los bosques nativos están en proceso de degradación debido a la deforestación.

Ante esto surge la necesidad de elaborar nuevas políticas y fortalecer las ya existentes, porque, a partir del análisis del marco jurídico de los tres países, se detectó que, por un lado, en cuanto a las fortalezas, los tres casos buscan el uso y aprovechamiento sostenible, así como la conservación y protección de los bosques.

Sin embargo, Argentina es el único caso que cuenta con una Ley de Presupuestos 
Mínimos, que trata única y específicamente a los bosques nativos y los servicios ecosistémicos que estos proveen. No obstante, los casos de Colombia y Perú son los únicos que refieren y tratan el tema de la deforestación en sus bosques y que, a su vez, regulan el incentivo de pago por servicios ambientales o mecanismos de retribución por servicios ecosistémicos.

Por otra parte, en cuanto a las debilidades, en los tres casos se observa una profusa normativa fragmentada, que regula solo aspectos parciales de los bosques. Particularmente, en el caso de Argentina no se ha avanzado en la sanción de una normativa específica respecto de los servicios ecosistémicos de forma integral. $\mathrm{Ni}$ tampoco (en el mismo caso) se ha avanzado en el pago por servicios ambientales o en alguna norma que regule la deforestación. Y, finalmente, dentro del marco jurídico de los tres países seleccionados, el enfoque social es muy limitado, ya que, por el contrario, se ha enfocado más en lo ambiental. Por lo que a partir de las fortalezas y debilidades detectadas, se observa que en ninguno de los casos se logra una protección jurídica total de estos ecosistemas. Es por eso que se debe fortalecer la normativa en materia de bosques y de sus servicios ecosistémicos para asegurar su permanencia en el tiempo, garantizándoselos a las generaciones presentes y, principalmente, a las futuras.

Es decir, resulta crucial la protección de estos ecosistemas forestales no solo para proteger la biodiversidad que habita en ellos, sino también para prevenir las inundaciones, la erosión, los incendios; evitar el avance de la desertificación; asegurar la calidad del agua que consumimos y del aire que respiramos, entre otras cosas. Es por todo lo mencionado que resulta indispensable su protección, fundamentalmente a partir de un marco jurídico que asegure su conservación y preservación.

\section{Referencias}

Balvanera, P., Castillo, A., Lazos, E., Caballero, K., Quijas, S., Flores, A. C., Galicia, C., Martínez, L., Saldaña, A., Sánchez, M., Maass, M., Ávila, P., Martínez, Y., Galindo, L., \& Sarukhán, J. (s.f.). Marcos conceptuales interdisciplinarios para el estudio de los servicios ecosistémicos en América Latina. Valoración de servicios ecosistémicos. Conceptos, herramientas y aplicaciones para el ordenamiento territorial (pp. 38-67). Universidad Nacional Autónoma de México.

Balvanera, P., \& Cotler, H. (2007). Acercamientos al estudio de los servicios ecosistémicos. Gac. Ecol., 84-85, 8-15. Secretaría de Medio Ambiente y Recursos Naturales; México.

Camacho Valdez, V., \& Ruiz Luna, A. (2012). Marco conceptual y clasificación de los servicios ecosistémicos. Rev. Biociencias, 1(4), 3-15. http:// revistabiociencias.uan.mx/index.php/BIOCIENCIAS/article/view/19

Diario Oficial El Peruano (2014). Ley de Mecanismos de Retribución por Servicios Ecosistémicos [Ley 30.215]. http://www.minam.gob.pe/ 
wp-content/uploads/2014/06/ley_302105_ MRSE.pdf

Ferro, M., \& Minaverry, C. M. (2019). Aportes normativos, institucionales y sociales a la gestión del agua y el enfoque ecosistémico en la cuenca del río Luján, Argentina. Rev. Derecho, 20, 25-55.

Fundación Vida Silvestre (s.f.) ¿Por qué son importantes los bosques? https://www. vidasilvestre.org.ar/nuestro_trabajo/legislacion_ambiental22/bosqu es/nos_ plantamos_por_los_bosques/_por_que_ son_importantes_los_bosques_/

Howarth, R. B., \& Wilson, M. A. (2002). Discourse-based Valuation of Ecosystem Services: Establishing Fair Outcomes through Group Deliberation. Ecol. Econ., 41(3), 431-443.

Minaverry, C. M. (2014). La normativa de los servicios ambientales en Sudamérica. Propuestas para una gestión sustentable. $\mathrm{Ob}$ serv. Medioamb., 17, 341-359. issn: 1139-1987. http://dx.doi.org/10.5209/rev_OBMD.2014. v17.47198

----- (2017). ¿Avances o retrocesos? La evolución de los paradigmas sobre gestión ambiental en relación con la normativa y jurisprudencia sobre servicios ecosistémicos en América Latina. Lex Soc., 7(1).

---- (2017). Progresos e inflexiones en el desarrollo normativo para la protección de los bosques nativos en el ámbito provincial argentino y uruguayo. Rev. Fac. Der., 42, 181209.

----- (2020). El reconocimiento de los servicios ecosistémicos culturales en el ámbito cientí- fico del derecho ambiental y de las ciencias sociales. Aplicación de sus contribuciones al caso de la protección jurídica de los bosques nativos en Norpatagonia argentina y chilena. Rev. lex, 18(25), 107-138.

Ministerio de Ambiente y Desarrollo Sostenible (2017). Por el cual se establece el pago por servicios ambientales y otros incentivos a la conservación [Decreto-Ley 870]. https:// www.minambiente.gov.co//images/normativa/app/decretos/870-ba.pdf

-----. Segundo Inventario Nacional de Bosques Nativos. https://www.argentina.gob.ar/ambiente/bosques/segundo-inventario-nacionalbosques-nativos

----- (2021). “Se presentó el Segundo Inventario Nacional de Bosques Nativos de Argentina”. https://www.argentina.gob.ar/noticias/ se-presento-el-segundo-inventarionacional-de-bosques-nativos-de-argentina

----- (s.f.) Manejo sostenible de bosques. https:// www.argentina.gob.ar/ambiente/tierra/ bosques-suelos/manejosostenible-bosques

Ministerio de Justicia (2002). Ley General del Ambiente [Ley 25.675]. http://servicios.infoleg.gob.ar/infolegInternet/anexos/7500079999/79980/norma.htm

---- (2016). Decreto Legislativo que establece medidas para la lucha contra la tala ilegal [Decreto Legislativo n.o 1220]. http://www.minam.gob.pe/wp-content/ uploads/2017/04/Decreto-LegislativoN\%C2\%B0-1220.pdf

Ministerio de Justicia y Derechos Humanos (1948). Ley de Defensa, Mejoramiento y Am- 
pliación de Bosques [Ley 13.273]. http://servicios.infoleg.gob.ar/infolegInternet/anexos/30000-34999/30713/norma.htm

----- (2007). Ley sobre Presupuestos Mínimos de Protección Ambiental de los Bosques Nativos [Ley 26.331]. http://servicios.infoleg.gob.ar/infolegInternet/anexos/135000139999/136125/norma.htm

Ministerio del Ambiente del Perú (2016). La conservación de bosques en el Perú (20112016). https://sinia.minam.gob.pe/documentos/conservacion-bosques-peru-2011-2016

Ministerio del Medio Ambiente (1996). Régimen de aprovechamiento forestal [Decreto 1791]. https://www.acnur.org/fileadmin/Documentos/BDL/2008/6517.pdf

Organización de las Naciones Unidas (ONU) (2003). Evaluación de los Ecosistemas del Milenio.

Organización de las Naciones Unidas para la Alimentación y la Agricultura (FAO) (2005). Análisis comparativo entre la legislación forestal vigente y la propuesta de la nueva Ley forestal, de las Áreas Protegidas y de la Vida Silvestre. Proyecto "Apoyo a la operacionalización del marco jurídico forestal y del Pronafor de Honduras". Programa de Cooperación FAO/Países Bajos (fnpp). http://www. fao.org/forestry/12927- 037222a94fb67c52726d46a319d87d85d.pdf

Ruiz Pérez, M., García Fernández, C., \& Sayer, J. A. (2007). Los servicios ambientales de los bosques. Ecosistemas, 16(3), 81-90. Asociación Española de Ecología Terrestre; Alicante, España.
Secretaría de Ambiente y Desarrollo Sustentable (2005). Primer Inventario Nacional de Bosques Nativos. https://www.argentina. gob.ar/sites/default/files/primer_inventario_nacional_-_informe_nacional_1.pdf

Secretaría General de la Alcaldía Mayor de Bogotá, D. C. (2015). Por la cual se expide el Plan Nacional de Desarrollo 2014-2018 “Todos por un nuevo país” [Ley 1753]. https://colaboracion.dnp.gov.co/CDT/Normograma/ Ley\%201753\%20de\%20201 5.pdf

Servicio Nacional Forestal y de Fauna Silvestre (Serfor) (2015). Ley Forestal y de Fauna Silvestre [Ley 29.763]. https://www.minam. gob.pe/wp-content/uploads/2017/04/ Ley-N\%c2\%b0- 29763.pdf

Servicio Nacional de Sanidad y Calidad Agroalimentaria (Senasa) (2014). Bosques argentinos, actividad forestal y economías regionales. http://www.senasa.gob.ar/senasacomunica/ infografias/bosques-argentinos-actividad-forestal-y-economiasregionales. 